\title{
Article
}

\section{Phages Enter the Fight against Colorectal Cancer}

Kannen, Vinicius, Parry, Lee and Martin, Francis L

Available at http://clok.uclan.ac.uk/30979/

Kannen, Vinicius, Parry, Lee and Martin, Francis L ORCID: 0000-0001-85624944 (2019) Phages Enter the Fight against Colorectal Cancer. Trends in Cancer, 5 (10). pp. 577-579.

It is advisable to refer to the publisher's version if you intend to cite from the work. http://dx.doi.org/10.1016/j.trecan.2019.08.002

For more information about UCLan's research in this area go to http://www.uclan.ac.uk/researchgroups/ and search for < name of research Group>.

For information about Research generally at UCLan please go to http://www.uclan.ac.uk/research/

All outputs in CLoK are protected by Intellectual Property Rights law, including Copyright law. Copyright, IPR and Moral Rights for the works on this site are retained by the individual authors and/or other copyright owners. Terms and conditions for use of this material are defined in the policies page.

\section{CLoK}

Central Lancashire online Knowledge www.clok.uclan.ac.uk

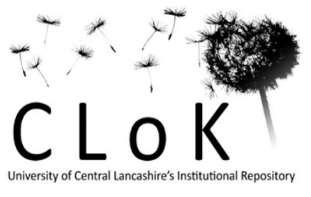


1 Phages enter the fight against colorectal cancer

3 Vinicius Kannen ${ }^{1}$, Lee Parry², Francis L. Martin ${ }^{3}$

$4 \quad{ }^{1}$ Department of Biomedical and Molecular Sciences, Queen's University, Kingston,

5 Canada

6 2European Cancer Stem Cell Research Institute, Cardiff School of Biosciences, Cardiff

7 University, Cardiff, UK

$8{ }^{3}$ School of Pharmacy and Biomedical Sciences, University of Central Lancashire,

9 Preston, UK

$23{ }^{*}$ Correspondence: vkc@queensu.ca (V. Kannen) 


\section{Summary}

Intestinal microbiota undergo significant changes in colorectal cancer (CRC). Zheng et al. (Nature Biomed Eng. 2019) observe detrimental overpopulation of Fusobacterium nucleatum ( $F$. nucleatum) in mice and patients, suppressing the beneficial butyrateproducing Clostridium butyricum. Phage-guided irinotecan-loaded dextran nanoparticles promote release of bacterial-derived butyrate, while $F$. nucleatum and $\mathrm{CRC}$ cells are eliminated. These findings describe a possible novel therapeutic strategy for CRC.

\section{Keywords}

Bacteriophage; colorectal cancer; chemotherapy; microbiota

Colorectal cancer $(\mathrm{CRC})$ is one of the three leading causes of cancer-related deaths worldwide. This multi-factorial and multi-stage disease impacts the life of millions of newly-diagnosed patients annually. Due to disease recurrence and metastasis, clinical management of CRC patients is associated with high healthcare costs. Currently, novel therapeutic strategies are urgently needed. A recently reported alternative approach has been to treat patients with bacteriophage (phage) for different pathological conditions. These bacteria-killing viruses can be classified as lytic or temperate units, as they either undergo lytic or lysogenic cycles, respectively. Although both events lead to bacterial destruction, lysogenic events require that viral and bacterial genomes integrate and replicate during the virus's dormant phase [1-4].

Recently, Zheng et al. [1] identified that CRC patients and $\mathrm{Apc}^{\mathrm{Min} /+}$ mice exhibit a significant increase of Fusobacterium nucleatum ( $F$. nucleatum), which is typically found 
47 in oral and nasal cavities. As an alien bacterial species to the intestines, F. nucleatum

48 produces immune blocking agents and competes with the beneficial butyrate-producing Clostridium butyricum (C. butyricum) units, thus promoting CRC development and 50 enhancing chemotherapy resistance. In human saliva, the authors isolated a temperate 51 phage capable of specialized and targeted killing of $F$. nucleatum without impacting the 52 C. butyricum population. From this, the authors went on to treat piglets with phage-guided dextran nanoparticles. Their experiments reveal that in response to this therapeutic strategy, minimal changes occur in vital metabolic or immunological functions. They further developed a phage-guided biotic-abiotic hybrid nanosystem that could increase the chemotherapeutic potency of irinotecan against CRC cells whilst also selectively killing the F. nucleatum population and allowing the butyrate-producing bacteria to expand in numbers at the same time. Phages have a long history of usage with varying success (Figure 1). Careful consideration should thus be given to this exciting discovery, as this new therapeutic strategy of administering phage-guided irinotecan-loaded dextran nanoparticles may impact CRC treatment in coming years.

It should be appreciated that the composition of the intestinal phage population has been reported to be altered at different stages of CRC development. Parabacteroides phage $Y Z-2015 b$ units undergo an exponential increase from early to late CRC stages [5]. In keeping with this idea, adherent invasive Escherichia coli promotes tumor growth in $A P C^{\mathrm{Min} /+}$ mice, while phage treatment has been found capable of increasing survival and reducing tumor growth in these bacteria-infected mice. However, elimination of cancer-causing bacteria via phages appears to worsen inflammatory bowel disease, as they alter the intestinal immunity. Studies have found lower phage levels in responsive 
70 rather than non-responsive ulcerative colitis patients who received fecal microbiota

71 transplantation since phages increase the release of interferon $y$ (IFN- $\gamma$ ) in this non-

72 responders group [2]. Another study further reveals that colitis changes the intestinal

73 phage population toward a stochastic state of dysbiosis in mice and humans [3].

74 Moreover, intestinal inflammation has been shown to promote bacterial pathogenic

75 evolution through a disease-driven transfer of temperate phages, as it supports the expression of phage promoter Tum, free phage production and transfer, and bacterial

77 SOS response [4]. Collectively, these findings should focus our attention on the fact that 78 phage therapy may lead to unforeseeable side effects in humans.

Treating humans with bioengineered phages has, however, been proven a 80 promising therapeutic strategy in cases where traditional approaches have failed.

81 Successfully, Spencer and colleagues applied a three-phage cocktail in a teenager

82 patient who underwent bilateral lung transplantation but had a drug-resistant bacterial 83 infection. Following 121 days of phage treatment, this patient exhibited a favorable clinical 84 improvement, including enhanced liver function and significant resolution of infection sites 85 [6]. Another exciting investigation involved the use of engineered virus-like particles with 86

87 enabled the specific elimination of human pluripotent stem cells by 5 -fluorocytosine without impacting on the non-target differentiated cellular population [7].

Although the idea of Zhang and colleagues to block CRC development by enhancing bacteria-producing butyrate through bioengineering-related methods is indeed

91 remarkable and mechanistically attractive [1], there is a wealth of evidence countenancing

92 caution to be considered. For instance, dextran has been chemically bound to 2- 
93 nitroimidazole $(\mathrm{NI})$. $\mathrm{NI}$ can be metabolized into bioreductive species that block DNA 94 synthesis and damage several intracellular targets in both eukaryotic and prokaryotic 95 cells, leading to nonselective cell death. Compounds of this class have indeed been 96 suggested to act as radiosensitisers [8]. This illustrates the carcinogenic potential that this 97 class of compounds have been reported to possess [9]. On the other hand, Park et al. 98 report that Gram-positive commensal bacteria control the intestinal epithelial cell turnover 99 by releasing short-chain fatty acids [10]. Stappenbeck and colleagues then revealed that 100 while the short-chain fatty acid butyrate is essential to colonocytes' metabolism, it inhibits 101 forkhead box o3 (FOXO3) transcriptional activity impairing proliferation in the colonic stem 102 cell niche [11]. However, Merchant and colleagues indicated that in Apc mutant stem 103 cells, in which $\beta$-catenin signaling is high, butyrate-dependent regulation through zinc 104 finger DNA-binding protein 89 (ZBP-89) promotes the development of early CRC stages 105 [12]. These facts should stimulate a thoughtful debate on the diverse effects of butyrate 106 on stem cells in physiological or malignant colon conditions, as it could significantly impact 107 cancer relapse and the patient's overall survival.

We believe that the findings of Zheng et al. embrace a promising and feasible 109 therapeutic strategy for CRC patients, as it provides a significant advancement in 110 therapies that strengthens the body's anticancer mechanisms with chemotherapy. 111 Further development should shed light on how this type of treatment reinvigorates the 112 anti-tumor immunity and impacts the behavior of cancer stem cells in CRC cases. 
115 1. Zheng, D.-W. et al. (2019) Phage-guided modulation of the gut microbiota of mouse 116 models of colorectal cancer augments their responses to chemotherapy. Nature 117 Biomedical Engineering.

118 2. Gogokhia, L. et al. (2019) Expansion of Bacteriophages Is Linked to Aggravated 119 Intestinal Inflammation and Colitis. Cell Host Microbe 25 (2), 285-299 e8.

120 3. Duerkop, B.A. et al. (2018) Murine colitis reveals a disease-associated bacteriophage 121 community. Nature Microbiology 3 (9), 1023-1031.

122 4. Diard, M. et al. (2017) Inflammation boosts bacteriophage transfer between Salmonella 123 spp. Science 355 (6330), 1211-1215.

124 5. Nakatsu, G. et al. (2018) Alterations in Enteric Virome Are Associated With Colorectal 125 Cancer and Survival Outcomes. Gastroenterology 155 (2), 529-541 e5.

126 6. Dedrick, R.M. et al. (2019) Engineered bacteriophages for treatment of a patient with 127 a disseminated drug-resistant Mycobacterium abscessus. Nature Medicine 25 (5), 730 128733.

129 7. Rampoldi, A. et al. (2018) Targeted Elimination of Tumorigenic Human Pluripotent 130 Stem Cells Using Suicide-Inducing Virus-like Particles. ACS Chem Biol 13 (8), 23291312338.

132 8. Meissner, R. et al. (2019) Low-energy electrons transform the nimorazole molecule 133 into a radiosensitiser. Nat Commun 10 (1), 2388.

134 9. Adil, M. et al. (2018) Association of Metronidazole with Cancer: A Potential Risk Factor 135 or Inconsistent Deductions? Curr Drug Metab 19 (11), 902-909.

136 10. Park, J.H. et al. (2016) Promotion of Intestinal Epithelial Cell Turnover by Commensal 137 Bacteria: Role of Short-Chain Fatty Acids. PLoS One 11 (5), e0156334. 
138 11. Kaiko, G.E. et al. (2016) The Colonic Crypt Protects Stem Cells from Microbiota-

139 Derived Metabolites. Cell 165 (7), 1708-1720.

140 12. Ocadiz-Ruiz, R. et al. (2017) ZBP-89 function in colonic stem cells and during

141 butyrate-induced senescence. Oncotarget 8 (55), 94330-94344.

\section{Figure legend}

144 Figure 1 - A century of phage research and therapy. Following the discovery of phages 145 at the beginning of the $20^{\text {th }}$ century, this class of bacteria-killing viruses was successfully 146 applied in the treatment of a broad range of bacterial infections. However, unfortunate 147 events impaired the evolution of such therapies in Western countries for more than a half148 century. The urgent emerging need for treatments for extensively drug-resistant or totally 149 drug-resistant bacteria has led the scientific community to revisit the potential application 150 of phages in treating a wide range of conditions. This has been expanded to include 151 cancer. 